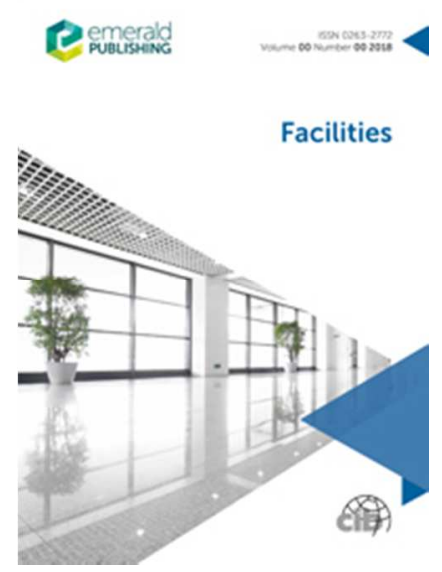

Improvement of the Inspection-Repair Process with Building Information Modelling and Image Classification

\begin{tabular}{|r|l|}
\hline Journal: & Facilities \\
\hline Manuscript ID & f-01-2018-0005.R2 \\
\hline Manuscript Type: & Original Article \\
\hline Keywords: & $\begin{array}{l}\text { Automation, Building information modelling, Inspection-repair process, } \\
\text { Digital 3D model, Operation and maintenance, facility management }\end{array}$ \\
\hline \multicolumn{2}{|l}{} \\
\hline
\end{tabular}




\title{
Improvement of the Inspection-Repair Process with Building Information Modelling and Image Classification
}

\begin{abstract}
Purpose-Automated technologies have been applied to facility management (FM) practices to address labour demands of, and time consumed by, inputting and processing manual data. Less attention has been focused on automation of visual information, such as images, when improving timely maintenance decisions. This study aimed to develop image classification algorithms to improve information flow in the inspection-repair process through building information modelling (BIM).
\end{abstract}

Design/methodology/approach - To improve and automate the inspection-repair process, image classification algorithms were employed to connect images with a corresponding image database in a BIM knowledge repository. QR code decoding and Bag of Words were chosen to classify images in the system. Graphical user interfaces (GUIs) were developed to facilitate activity collaboration and communication. A pilot case study in an inspection-repair process was applied to demonstrate the applications of this system.

Findings - The system developed in this study associates the inspection-repair process with a digital three dimensional (3D) model, GUIs, a BIM knowledge repository and image classification algorithms. By implementing the proposed application in a case study, the authors found that improvement of the inspection-repair process and automated image classification with a BIM knowledge repository (such as the one developed in this study) can enhance FM practices by increasing productivity and reducing time and costs associated with decision-making.

Originality/value - This study introduces an innovative approach that applies image classification and leverages a BIM knowledge repository to enhance the inspection-repair process in facility management practice. The system designed provides automated image-classifying data from a smart phone, eliminates time required to input image data manually, and improves communication and collaboration between FM personnel for maintenance in the decision-making process.

Keywords: automation, facility management, building information modelling, inspection-repair process, operation and maintenance, digital 3D model.

Paper type-Research paper 


\section{Improvement of the Inspection-Repair Process with Building Information Modelling and Image Classification}

\section{Introduction}

Facility management (FM) involves multitasking and complex processes, including environmental health and safety (EHS), space planning, security, building operation, repairing and managing emergencies. As the final and longest phase of a facility's lifecycle (Akcamete et al. 2010), FM requires day-to-day operation and maintenance (O\&M) activities to optimise performance of buildings, systems and equipment for building occupiers. Ineffective building maintenance poses significant safety risks to building occupiers and results in great economic losses for building owners.

A key problem in practical FM is that inspecting facilities, collecting data and assessing maintenance is labour-intensive, time consuming, and may yield inaccurate data with the potential to mislead management decisions. Management is required to track and control inspections and maintenance processes, as well as monitoring the real-time performance of a facility by accessing information such as facility conditions, specifications, maintenance history, insurance and warranty information to make decisions regarding repairs and maintenance. With manual, paper-based recording of information, data that is captured can differ from data that is entered. Due to the need for repeated collection of information, O\&M costs are relatively high, at around $60 \%$ or two-thirds of the cost incurred during the entire life cycle of a facility (Liu and Issa, 2016). The main reasons for low efficiency in existing O\&M practices include lack of an automated and economical approach for accurate data processing or up-to-date data repositories (Wetzel and Thabet, 2015; Lu and Lee, 2017). Digital technologies such as computer-aided facility management (CAFM), building information systems (BISs) and building information modelling (BIM) have been introduced into facility operation functions in recent years to tackle issues around real-time information collection and processing. Lavikka et al. (2017) suggest that digital building data allows for the creation of digital FM services that 'can reduce the building life-cycle costs, increase the performance of the building and increase the productivity of the users'. However, it is a challenge to integrate real-time inspection results from multiple data sources, in particular image sources, and measurement devices. There is increasing value in using images to provide visual data to identify the facility conditions of various building elements such as steel corrosion, wall cracks and painting in carpet, pine, timber and other elements that cannot be detected by sensors. Lu and Lee (2017) suggest that it would be more effective and economical in building management processes to generate an 'as-is' BIM model using image-based modelling methods. Another challenge is to recognise the inspected images and update them to the corresponding image database.

To enhance the effectiveness of inspection and maintenance decisions, this study aimed to use BIM to develop an efficient data management system to improve image data flow in the FM inspection-repair process. Specifically, three objectives were put forward: a) to develop a BIM knowledge repository that stores activity history, images and other information for convenient access; b) to apply algorithms in order to classify images to their content automatically; and c) to create user-friendly interfaces to facilitate collaboration between personnel.

This paper advances the existing FM process by incorporating QR codes with BIM, as well as image classification using Bag of Words. This study extends the application of image-based object recognition (Lazebnik et al. 2006) methods to include BIM in FM processes. Image data were collected through smart phones, integrated with BIM and analysed using deep-learning algorithms. The next section of this paper examines existing literature regarding BIM applications in FM. Methods of image classification and related knowledge including QR codes and Bag of Words will be reviewed. The third section describes the system design, including discussion of a pilot study testing a prototype system. Concluding remarks will be made at the end of the paper.

\section{Literature Review}

Regular inspections help to ensure that building facilities and equipment can perform their intended functions, improving productivity and reducing running costs. In addition, regular inspections ensure that the regulatory compliance of buildings and safety requirements of occupiers are met. Traditionally, building inspectors 
undertake a manual process to identify the condition of and defects in facilities, inputting inspection data manually. However, this process is time-consuming and costly (Hamledari et al. 2018). Missing or incorrect building information can arise in a manual process, reducing efficiency in decision-making processes in the O\&M, leading to significant delays in responding to emergencies and daily requests of building occupants (Kang and Choi, 2015). Moreover, unintended errors or accidents can result from uncertainties around recognition of existing building documents and inaccurate assumptions regarding building conditions (Hamledari et al. 2018). Problems with manual building inspection processes suggest that an automated inspection-repair system that can provide correct building information instantly would assist in FM decisionmaking. BIM is an emergent technology that provides a comprehensive platform in FM for up-to-date and accurate information.

\section{BIM in FM}

The use of BIM in FM enables lifecycle data management and monitoring of the performance of FM activities such as: developing preventive maintenance plans; commissioning, maintenance and servicing; space making; quality control; energy performance; managing emergencies; and deconstruction (Volk et al. 2014; Costin et al. 2013; Becerik-Gerber et al. 2012; Chen et al. 2011; Akcamete et al. 2010). The benefits of integrating BIM and FM include: reducing operational costs; reducing time needed for decision-making; improving documentation systems; facilitating collaboration and work flexibility; and simplifying information updates and clash detections (Aziz et al. 2016). Motamedi et al. (2014) illustrated that BIM models have object visualisation functions, allowing FM technicians to view facilities in 3D. Thus, the cognitive and perceptual reasoning skills of BIM models can solve maintenance problems.

Although the development of BIM-FM integration is evident, adoption of BIM in FM has not received as much attention as in earlier phases of design and construction (Akcamete et al. 2010). Becerik-Gerber et al. (2012) surveyed 77 FM groups on the potential utilisation of BIM applications in FM application areas. Results showed that $83 \%$ and $79 \%$ of BIM users used BIM in design and construction phases, respectively, while only $42 \%$ used BIM in FM. Parn et al. (2016) summarise three main challenges for FM-BIM integration: 1) data supplied in FM does not meet BIM data requirements; 2) FM personnel lack training in using the relevant technology; and 3) FM personnel do not understand how information within BIM can facilitate decision-making in FM. Between 2005 and 2015, the number of published papers on BIM-FM was lower than the number of published papers on all other categories of BIM (Santos et al. 2017), suggesting a lack of research on BIM in FM.

\section{Image-based methods and automatic recognition}

Researchers note advantages of image-based methods over laser-scanning-based methods, which are more expensive, and point clouds, which may produce noisy data or miss data altogether (Fathi et al. 2015). Imagebased methods improve visualisation and accuracy (Bhatla et al. 2012; Bosche et al. 2015; Dimitrov and Golparvar-Fard, 2014; Lu and Lee, 2017; Dai et al. 2012; Klein et al. 2012) and are effective and economical. However, image-based methods may require extra effort in terms of processing ( $\mathrm{Lu}$ and Lee, 2017).

Image-based recognition consists of five categories (Liu and Issa, 2014). The first category is featurerepresentation-based methods, which mainly focus on the feature extraction process. One application of this method is to conduct single sample per person (SSPP) classification (Ding et al. 2015). Shao et al. (2003) introduced a hyper-polyhedron with the adaptive threshold (HPAT) technique, which can be used for small building images. Suleiman et al. (2011) developed a technique to recognise facades in a photograph. An image taken with a camera may contain exact feature information as well as its geo-location information.

The wide-baseline-matching-based method is the second category of image-based recognition. This method discovers relationships and correspondences between the reference image and the querying image of the same scene. The wide-baseline-matching-based method is suited to highly-textured scenes. However, it cannot deal with poorly textured scenes ( $\mathrm{Lu}$ and Lee, 2017). The third category includes dimensionalityreduction-based methods, such as the linear-subspace method (LSM) and nonlinear dimensionality-reduction 
method (Zhang et al. 2009). The original feature in a high-dimensional space transfers into a much lowerdimensional space to eliminate redundant features and increase efficiency. Principal component analysis (PCA) and linear discriminant analysis (LDA) are the most typical and essential algorithms of an LSM. The fourth category consists of clustering-based methods that classify objects or patterns by grouping various features into different clusters. The intra-structure and semantic interrelationships of each cluster are considered as characteristics for object recognition (Lazebnik et al. 2006). Image-based 3D construction methods, the fifth category, create 3D point clouds of the target building using structure from motion (SfM) and a collection of overlapping images, instead of laser scanners. Image-based 3D construction methods have broad applications, ranging from the film industry to archaeological excavations (De Reu et al. 2014).

\section{Quick response (QR) code and Bag of Words}

Quick response (QR) codes are two-dimensional (2D) barcodes represented by symbols. The QR code itself stores huge amounts of information (up to 4,296 alphanumeric characters) which can be scanned and stored on a mobile device. Flexibility and versatility are the main advantages of using QR codes for storing and accessing information. A mobile device or smartphone is required to access the information from QR codes. Applications of QR codes have extended to the construction industry. One-dimensional barcodes have been used for data entry efficiency and cost cutting (Bell and McCullouch, 1988; Stukhart and Cook, 1990), labour management, productivity improvement (Blakey, 1990), construction equipment and materials tracking (Wakisaka et al. 2000), as well as electronic document management (Finch et al. 1996).

QR codes have been applied to integrate with other technologies (McCullouch and Lueprasert, 1994) including state-wide roadside signs with geographic information system (GIS) (Bell and Williams, 2003), automated data collection, materials management and control (Navon and Berkovich, 2005), automated systems for engineering deliverables such as drawings, reports and specifications (Shehab and Moselhi, 2005) and pedestrian real-time location and routing information with GIS (Saeed et al. 2010). QR codes have potential applications in the $\mathrm{BIM}+\mathrm{QR}$ code environment in construction site management, including operations, procedures and the schematisation of site equipment and temporary structures. These were investigated by Lorenzo et al. (2014). Less literature exists on the use of QR codes in facility management, except for research by Lin et al. (2014), who developed a facility management system that incorporated 2D barcodes and Radio-frequency identification (RFID) technologies. Lin et al. (2014) found that the system improved the effectiveness and convenience of maintenance management in a construction lab in Taiwan. In addition, Lavikka et al. (2017) conducted a case study on a medical centre where a QR code was applied to valves during the construction phase. Their study showed that QR codes can enhance FM by enabling fast information retrieval and increasing cooperation between parties. However, barcodes may not be accessible on pine or wall surfaces, and areas that require repairs - such as leaking water-may not have a barcode. An alternative way of collecting and accessing information on repairs and maintenance is needed.

Originally a representation used in document classification and later applied to conduct multi-class image classifications (Csurka et al. 2004), Bag of Words has been used in diverse fields. Examples include cancer detection, logo recognition, ancient coin classification and improper image filtering (Deselaers et al. 2008; He et al. 2016; Alfed et al. 2016; Anwar et al. 2013). Advantages of Bag of Words include its ease of use and low computational cost. The main steps required to implement Bag of Words for image classification include (Anwar et al. 2013; MathWorks n.d.; Vyas et al. 2016): extracting features from a set of training images; establishing a visual vocabulary, or Bag of Words, with a quantisation scheme; training a multi-class classifier with visual vocabularies as feature vectors; evaluating the quality of the classifier by testing the classifier with a test set of images; and applying the trained classifier to predict labels in images.

The literature review outlined above highlights how to develop an inspection-repair process facilitated by a management system using BIM and automated image classification. This study was intended to design a system that can enhance image-based FM processes and automatically link inspection images to their corresponding objects in the 3D BIM knowledge repository. This system was intended to address the challenge of automated information flows between the newly inspected data and the historical information 
stored in the BIM - in particular, image information that can be used for management decision-making. The following important research questions were investigated in this study: 1) what is the appropriate platform that can be used to store a large amount of image (that is, photographic) information for convenient access? 2) how can the images be classified automatically? and 3) how can effective communication be facilitated with inspectors, managers and repairmen?

\section{Research Method and System Design}

Little literature exists on automated systems that store data collected from inspections, identify data locations, classify data categories and communicate with repair decision automatically in FM. Motawa and Almarshad (2013) note that the current FM system fails to capture and retrieve detailed information and knowledge generated from building maintenance or operations. Photograph are acquired manually, which is impractical (Park et al. 2018). Due to the scarce literature in this field, the research methodology in this study involved an empirical study in which photo images were collected via smartphone from a bedroom and a kitchen. Methods including Bag of Words and a decoding system were chosen to classify images for automatic uploading and allocation to files stored in the BIM. For each element inspected, image classification algorithms were applied to identify the defect indicator, defect type, design inconformity and update the model type and property sets within the input BIM automatically. This allows relevant FM personnel to retrieve the images and conduct inspection-repair activities. Details of the inspection-repair automated system are described in the following four steps: 1) establishing an inspection-repair process and data collection; 2) design of system structure; 3) creation of a BIM knowledge repository; and 4) developing graphical user interfaces (GIUs) for depositing and managing images.

\section{Step 1: Establishment of inspection-repair process and data collection}

Typical building maintenance processes involve three main types of personnel: inspectors, managers and repairmen. Building inspectors inspect facilities on a regular basis, checking building objects and reporting facility conditions or damage to building managers if damaged objects are found. Building managers monitor the whole building maintenance process, receiving facility condition or damage reports from inspectors and assigning repairmen or other responsible people to take further action. Building repairmen are responsible for repairing damaged objects according to assignments they receive from managers. In addition, repairmen report back to building managers after completing their jobs. With the application of image-based management systems, a new inspection-repair process can be established (see Figure 1). An inspector inspects objects (for example, ceiling fans) in the physical environment and takes images of them using the camera built into their smartphone. Next, the inspector uploads the images to the system with automatic image classification, reporting any damage found. A facility manager then reviews these images and decides whether a repair is required. If a repair is needed, the manager assigns a repairman to repair those damaged objects, and the assigned repairman receives the tasks and repairs the damaged objects. After that, the repairman takes pictures of the repaired objects and uploads them to the system. Building managers can then review the images of objects that have been repaired. Each role corresponds to one kind of activity: 1) inspectors - inspections, 2) managers - assignment, and 3) repairmen - repair. 


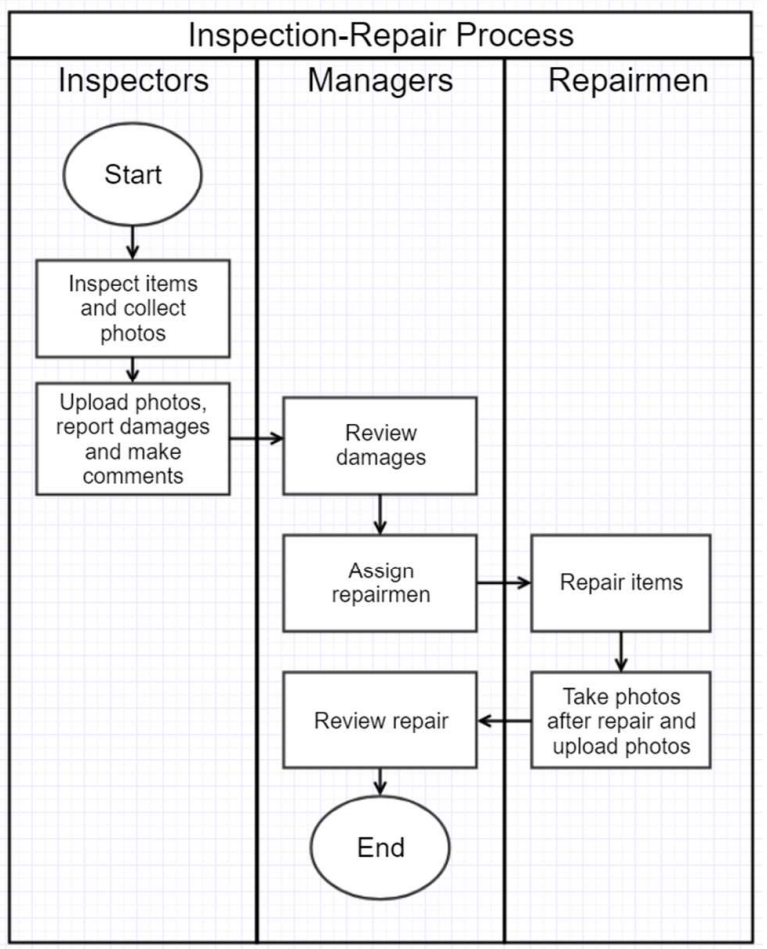

Figure 1. The Swim Lane Diagram of the inspection-repair process

\section{Step 2: Design of system structure}

First, data flows on the FM inspection to the maintenance decisions and the repair to the completion of the repair were identified. Next, personnel required for the process were established based on daily FM practical activities. Based on the established inspection-repair process, a management system was designed (see Figure 2). The main components of these systems are the BIM knowledge repository, including Navisworks, image and text files and image classification, including QR code decoding and classifiers with Bag of Words. MATLAB was used to implement image classification. Images and other information on activates are stored in different files in Windows. To connect these components, plugins are created with C\# and .NET, which are based on the Navisworks API. Windows Forms (WinForms) are associated with the plugins to provide GUIs for users.

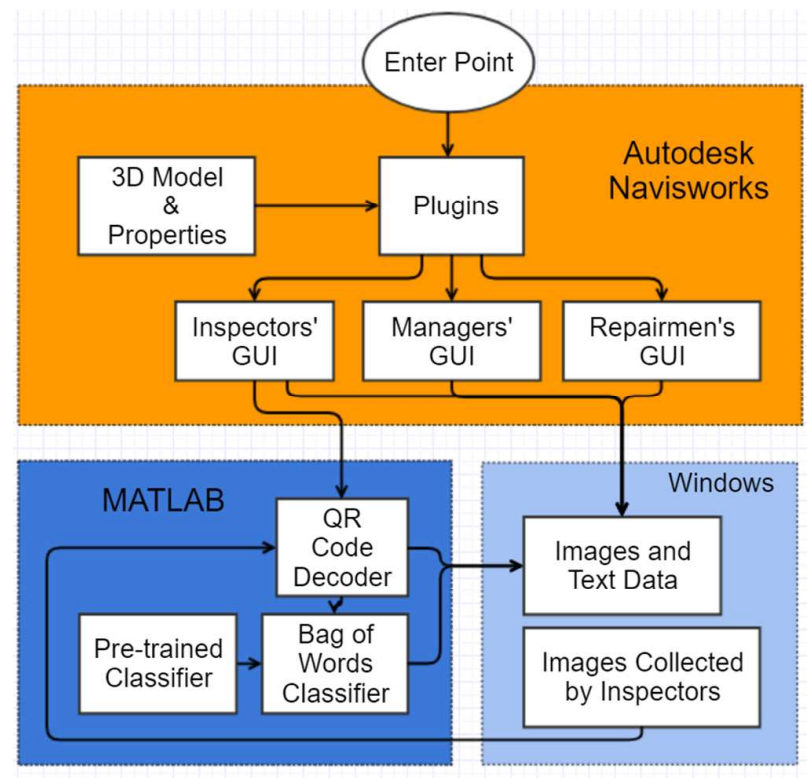

Figure 2. Major components of the system and their connections 


\section{Step 3: Creation of BIM knowledge repository}

The BIM knowledge repository stores all the information that is used in this system. This information is stored either in a Navisworks file or the Windows system (see Figure 3). The 3D model, object information and activity histories (for the inspection, assignment and repair) are saved in a Navisworks file. Other information, including images taken by inspectors or repairmen and user comments on activities, are stored in image files (.jpg or .png) or plain text files (.txt). All information is linked using Plugins, which can access and amend the information. All files stored in Windows are stored in a single root folder and its sub-folders (see Figure 4). The first layer of folders under the root folder is the classification of objects. Beneath that, each activity has its own folder. Inside the activity folders, the image files and a text file recording the information of this activity are sorted. Furthermore, an extra folder stores the information about each image in the text file. Since accessing these folders and files requires a name, the naming convention is required. In the text file, information is stored using markers and a comment is inserted between the markers. By using this format, information can be found accurately.

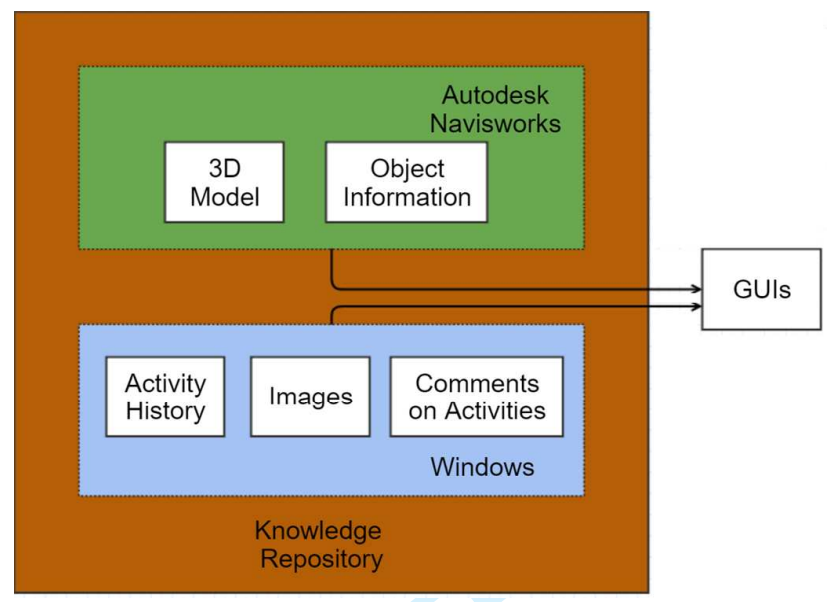

Figure 3. Components of knowledge repository

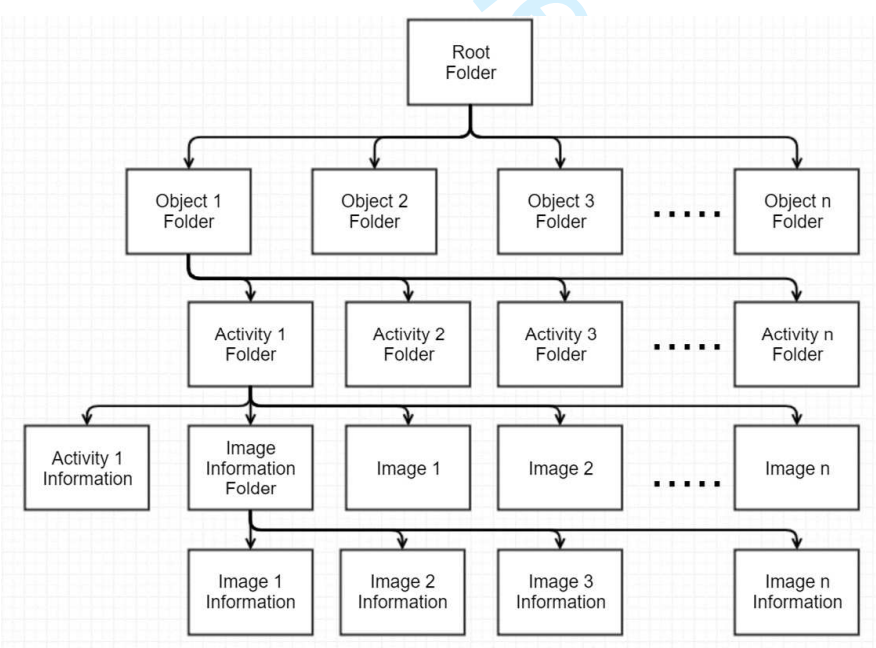

Figure 4. Structure of image and text data stored in Windows

The proposed BIM knowledge repository can store all information collected, including image data from inspections. The repository provides a flexible platform that allows image classification and identification of object locations (Park et al. 2018) implemented by other tools such as MATLAB. In addition, the repository allows the FM system to capture and retrieve detailed information and knowledge generated from building maintenance or operations (Motawa and Almarshad, 2013). BIM's object visualisation functions (Motamedi et al., 2014) enable FM managers and technicians to view facilities in 3D in maintenance decision-making and activities. 


\section{Step 4: Development of GUIs for depositing and managing images}

GUIs were built using WinForms from the .NET framework. Three separate sets of GUIs for inspectors, managers and repairmen were developed, each associated with plugins in Navisworks. The main functions of inspector GUIs include guiding inspectors to upload images from inspections, asking them to indicate damaged objects and leave comments, as well as providing a viewer in order to check the previous inspection. GUIs also help to inform managers whether damaged objects have been found and need to be repaired, enabling them to assign repairmen to repair these objects. As mentioned, building managers oversee the whole FM process. Therefore, their GUIs allow them to access all records of activities and comments. The GUIs for the repairmen facilitate the repair activity, which includes showing unfinished assignments from managers as well as images and comments from inspectors, and allow repairmen to upload images to the system.

\section{Empirical Study}

By following the system design procedure described in the previous section, the authors selected an apartment bedroom and kitchen to test the effectiveness of the developed system in the pilot study.

\section{a) Establishment of a BIM knowledge repository and collection of images}

Initially, a BIM 3D model of the facilities needed to be created. In this pilot study, a model of a bedroom connecting to a kitchen was created (Figure 5). The environment contained a fridge, microwave, coffee machine, sink, table, chair, bed, cabinet and a monitor. Some information was generated automatically when the model was created. Other information required input from users, such as information about manufacturers, purchase dates and warranty expiry date (see Figure 6). The root folders of each individual object, which were the first layer of files, needed to be established. After the BIM knowledge repository was established, the inspection-repair process was commenced. The process began with inspecting objects in the facilities and collecting photos of these objects with a smartphone. The images collected were uploaded to the system for automated classification with QR code decoding and Bag of Words.

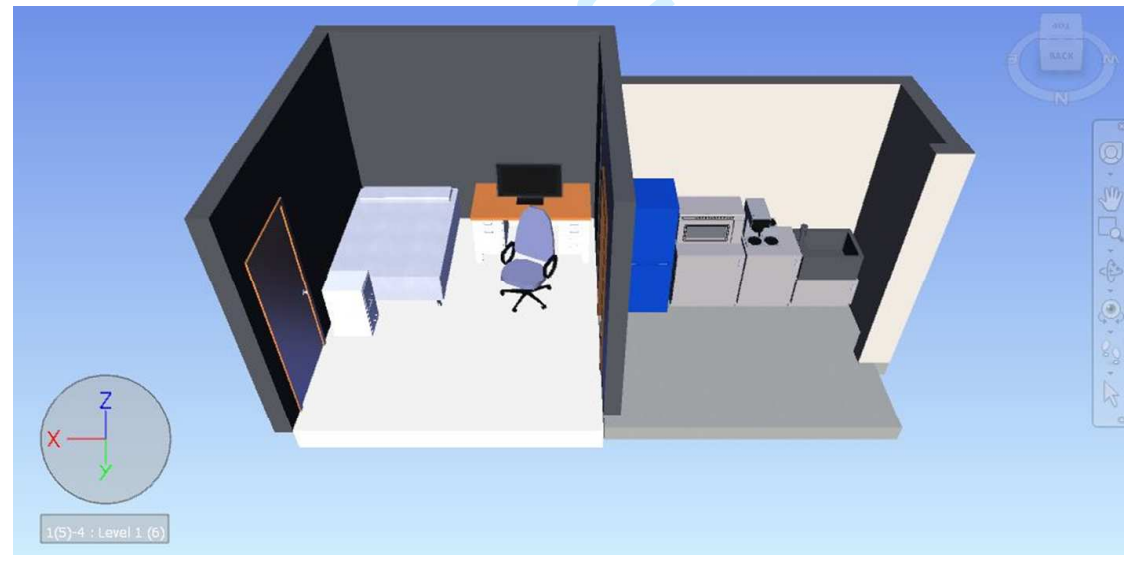

Figure 5. 3D Model of a bedroom and a kitchen

\section{b) Automatic image classification}

One of the key problems that this paper intended to solve is the inefficiency of information collection and management during building inspection processes. In order to solve this problem, an automatic process for classifying images was introduced, which links the images automatically to their corresponding objects in the 3D model. Each object was given a specific label as a form of identification, which was stored in its properties. QR codes and Bag of Words were chosen to classify images in the system —or, in other words, to identify the image label. QR codes were selected because the barcode technique has grown to include a wider range of applications including inventory and equipment management, commercial tracking, entertainment and transport ticketing, clinical trial management, and even biomedical imaging (Takran and Ylmaz, 2015; 
Su et al. 2014; Panyindee and Pintavirooj, 2013; Huang et al. 2011; GS1 2009). Evidence shows that each QR code stores unique information that can be identified and distinguished from other QR code data. To decode a QR code on an image, the following steps are executed: 1) edge detection for a binary images, 2) using the dilation process, 3) gaining black and white blocks, 4) scanning an image from top left to bottom right to position the code, 5) segmentation of the code, and 6) extracting the information from the code (Chang et al. 2007).

Both QR codes and image classifications with Bag of Words methods were used because neither method is $100 \%$ reliable. QR codes may not always be available for each object and they may not be readable by the system due to visibility and distortion. Image classification may malfunction occasionally, which may be caused by image resolution and clarity. Therefore, by combining these two methods, a more reliable classification process can be formed. The flow of the overall process is illustrated in Figure 6.

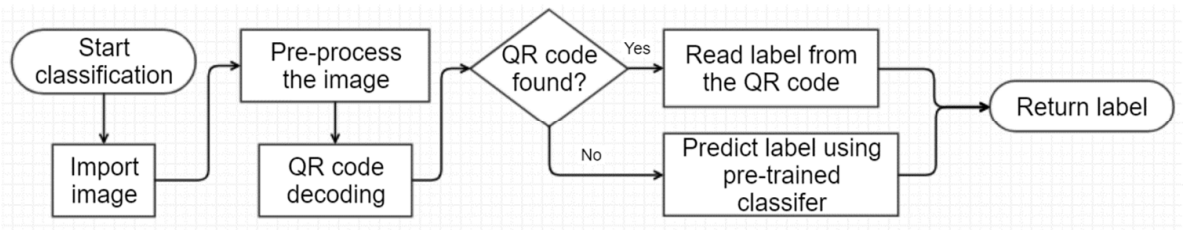

Figure 6. Flowchart of the process of identifying objects in an image

\section{c) Information retrieval using a $Q R$ code}

Lin et al. (2014) and Lavikka et al. (2017) have demonstrated how QR codes can be utilised for fast information retrieval from as-is objects. Meadati (2016) proposed an application of QR codes that automates information flow between BIM objects and as-is objects. Inspired by their studies, the QR code was chosen as one of the methods for automatic image sorting. By using a QR code, information about objects can be retrieved directly. To apply this method, physically pre-created QR codes with labels stored either on paper or paintings were attached to the objects. In this system, an open-source library called ZXing (short for Zebra Crossing) was used to decode QR codes from input images. According the International Organization for Standardization, four levels of error correlation can be selected when a QR code is created: L (seven percent), M (15\%), Q (25\%) and H (30\%). The error correlation percentage is proportional to the decoding capability when the QR code is partially invisible, but inversely proportional to the size of the data that can be stored. Through experiments, it has been found that the error correlation percentage is proportional to the successful reading rate when the $\mathrm{QR}$ code is distorted or small in images. Thus, to gain the highest reading rate, $\mathrm{QR}$ codes with error correction levels of H (30\%) were used. Since only a small amount of data needs to be stored in the QR code for this system, any limitations that arise due to data size will not be an issue.

\section{d) Image classification with Bag of Words}

Images can be taken by FM inspectors after inspections, or by repairmen completing facility maintenance. Without proper categorising, different types of images stored on BIM could become mixed up and disordered. By applying image classification, image data can be directed to the pre-coded file location automatically. This classification allows FM personnel to retrieve the correct information for maintenance purposes. As mentioned above, image classification has been applied to perform the automatic process along with a QR code. Bag of Words method was selected to conduct image classification because of its simplicity, maturity and practical performance (Nowak et al. 2006) in various applications. In addition, classifying different objects is one of the main objectives of this system, and recent studies have proven that Bag of Words is an effective and robust approach to object classification (Ali et al. 2016; Hannat et al. 2016; Vyas et al. 2016; Ergene and Durdu, 2017; Nguyen-Hoang et al. 2017; Unlu et al. 2017).

Other than Bag of Words, the convolutional neural network (CNN) was considered for conducting image classification. However, CNN was not chosen in this work. The architecture of CNN consists of multiple convolutional and max-pooling layers, followed by several fully connected layers and an output layer at the 
end (Abdelfattah, 2017). Although CNN is prominent for its high accuracy (Gupta, 2017; MathWorks n.d.), its disadvantages are obvious: CNN requires large sets of label data and a powerful GPU to be implemented effectively (Han et al. 2017). Both requirements restrict the use of this system. Therefore, CNN was ruled out.

As mentioned in the previous section, a classifier must be trained before it can be used to classify other images, and training sets of images of each category must be taken in advance. In order to train a classifier using Bag of Words, MATLAB and its Computer Vision toolbox (MathWorks n.d.) were used in this system. When images for training sets are ready, the first step was to extract features from these images. Dense sampling was used to extract features in this system, by splitting images with "grip", which shows suitability in indoor environments (Nowak et al. 2006). The density of sampling was set to eight pixels per grid step, which is the density most commonly used in other relevant studies (Ionescu et al. 2014). After that, feature descriptors of SURF were used to represent these samples as features. SURF was chosen because it is faster than STIF when processing and performs as well as STIF (Panchal et al. 2013).

After the extraction of features, the $K$-means clustering algorithm was applied to merge similar features into 500 clusters (that is, visual words). Together, these visual words eventually form a codebook. If features are treated as data points, a cluster consists of its centroid and data points that are closest to the centroid. According to $\mathrm{Wu}$ (2012), the clustering process of $K$-means is as follows. Initially, $K$ centroids are chosen in random locations, where $K$ is the number of clusters, and is set to 500 in this system. Data points are then assigned to their nearest centroid. Next, centroids are updated to have minimum distances to their data point, while data points remain unchanged. Data points may change their cluster due to updates to centroids. The previous step is repeated until no data points change its cluster between iteration, or the limit of 100 iterations is reached. If $x$ is the data to be clustered, $K$-means can be expressed with the following formula:

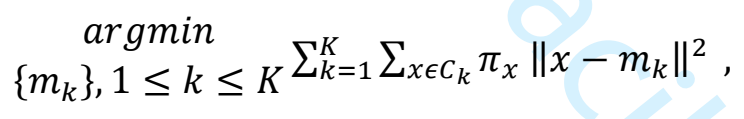

where $m_{k}$ is the centroid of cluster $C_{k}$, and

$$
m_{k}=\sum_{x \in C_{k}} \frac{\pi_{x} x}{n_{k}}
$$

$K$ is the number of clusters, $\pi_{x}$ is the weight of $x$, and $n_{k}$ is the number of data objects assigned to cluster $C_{k}$.

Once the codebook is completed, histograms were generated of the visual word frequency for each input image. In total, 500 clusters were assigned. Therefore, the codebook contained 500 visual words. When all histograms were attained, the error-correcting output codes (ECOC) method was used along with the binary support vector machine (SVM) to train a multi-class classier. The positive and negative samples came from the histogram of visual words.

A testing set of images can be used, with a confusion matrix used to quantify the set's performance. Next, the classifier can be applied to classify imported images after the classifier is trained. Then, the classifier can be used to classify other images by repeating the same steps above on new images.

Through experiments, it was found that the classifier has a greater level of performance with low resolution images than with high resolution images (numerical results will be explained in the Results section below). Therefore, the resolution of images to be used in this system was decided to be approximately 91,875 pixels ( $245 \times 375)$. Current smartphones often capture images at a resolution of $2448 \times 3264$ or higher, making it necessary to resize images before applying the classifier. In order to resize images to a resolution of 91,875 pixels, regardless of their original resolution, a simple formula was used (Formula 3). After the shrinking scale was computed, input images were shrunk by the shrinking scale amount on both horizontal and vertical axes, diminishing both directions by the same amount to avoid distortion in images.

$$
\text { Shrinking scale }=\frac{\sqrt{245 \times 327}}{\sqrt{\text { The number of pixels of the original image }}}
$$


Once the images were successfully classified, a form was created (Figure 7) where images could be reviewed, and damaged objects could be indicated. Comments could be left to progress the decision-making process to the next step.

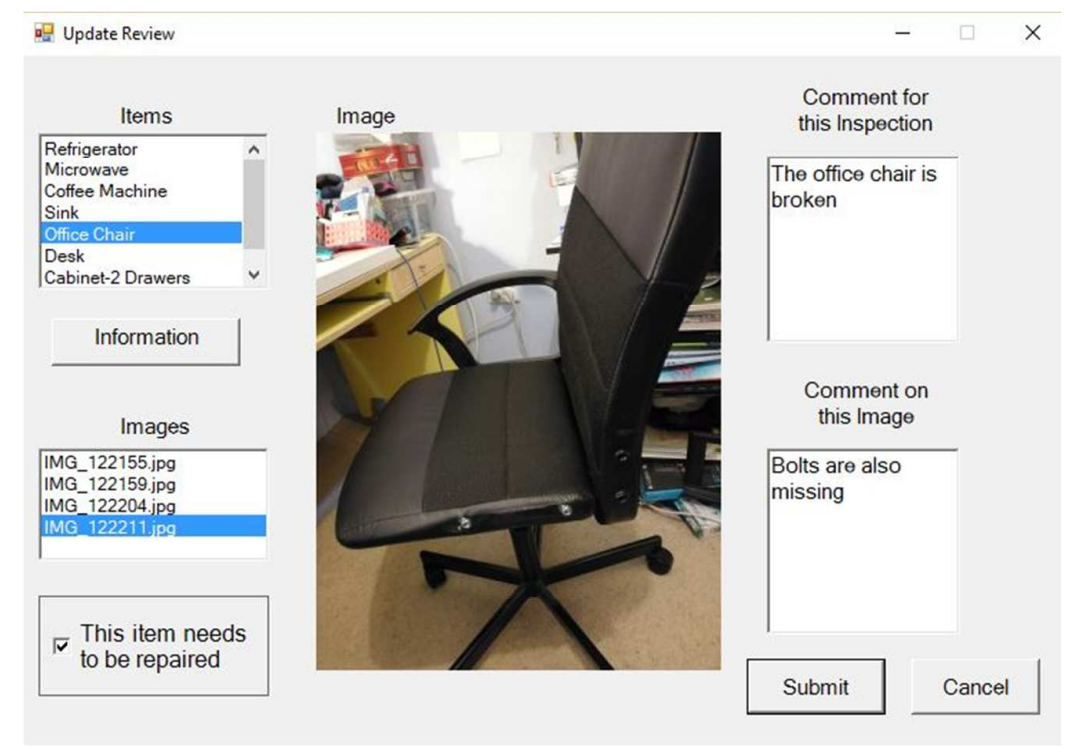

Figure 7. Interface for inspectors

\section{e) Decision-making, repair and review}

Next, a warning appears on the manager's interface and inspection results are submitted. Images of the damaged object are filtered out quickly and reviewed. Since the handle of the chair was missing, the decision was made to assign a repairman to repair it. After the chair was repaired, photos were taken and uploaded to the system and comments were left. The repair activity then appears in the manager's interface, which can be reviewed when needed.

\section{Empirical Results}

\section{Accuracy and processing time}

An experiment was conducted to research the relationship between resolution of input images and accuracy of classification, as well as resolution and processing time. In the experiments, six classes were defined, namely refrigerator, microwave, coffee machine, sink, chair and monitor. 10 photos were taken for each class, forming a training with total 60 images. These images were resized for training into five classifiers, from 245 x 327 to 1224 x 1632 pixels. Trained classifiers were tested on other 48 images, with eight in each class. The results tabulated in Table 1 show that accuracy was higher when training and testing images had similar resolution. By comparing the diagonal cell on the table, where training images and testing images have the same resolution, it can be found that accuracy is inversely proportional to the resolution of images within the testing range, with a maximum of $98 \%$. However, an exception can be seen in the cell in the second row and third column, which reached $100 \%$. 
Table 1. Accuracy of Bag of Words classifiers trained on 60 images and tested on 48 images with different resolutions

\begin{tabular}{|l|l|l|l|l|l|}
\hline Accuracy (\%) & $\begin{array}{l}\text { Trained with } \\
\mathbf{2 4 5} \times \mathbf{3 2 7}\end{array}$ & $\begin{array}{l}\text { Trained with } \\
\mathbf{4 9 0} \times \mathbf{6 5 3}\end{array}$ & $\begin{array}{l}\text { Trained with } \\
\mathbf{7 3 5} \times \mathbf{9 8 0}\end{array}$ & $\begin{array}{l}\text { Trained with } \\
\mathbf{9 0 8} \times \mathbf{1 3 0 6}\end{array}$ & $\begin{array}{l}\text { Trained with } \\
\mathbf{1 2 2 4} \mathbf{x} \mathbf{1 6 3 2}\end{array}$ \\
\hline $\begin{array}{l}\text { Tested on } \\
245 \times 327\end{array}$ & $98 \%$ & $100 \%$ & $93 \%$ & $80 \%$ & $79 \%$ \\
\hline $\begin{array}{l}\text { Tested on } \\
490 \times 653\end{array}$ & $84 \%$ & $93 \%$ & $93 \%$ & $93 \%$ & $85 \%$ \\
\hline $\begin{array}{l}\text { Tested on } \\
735 \times 980\end{array}$ & $73 \%$ & $83 \%$ & $87 \%$ & $91 \%$ & $87 \%$ \\
\hline $\begin{array}{l}\text { Tested on } \\
908 \times 1306\end{array}$ & $72 \%$ & $77 \%$ & $76 \%$ & $83 \%$ & $83 \%$ \\
\hline $\begin{array}{l}\text { Tested on } \\
1224 \times 1632\end{array}$ & $68 \%$ & $70 \%$ & $77 \%$ & $82 \%$ & $80 \%$ \\
\hline $\begin{array}{l}\text { Tested on } \\
2448 \times 3264\end{array}$ & $30 \%$ & $30 \%$ & $53 \%$ & $70 \%$ & $72 \%$ \\
\hline
\end{tabular}

Processing time (see Table 2), is inversely proportional to the number of pixels of each image, which is also a linear relationship. Results from both tables show that low resolution images should be used for this system.

Table 2. Processing time of classifiers trained on 60 images and tested 48 images with different resolution

\begin{tabular}{|l|l|l|l|l|l|}
\hline $\begin{array}{l}\text { Time } \\
\text { (second/image) }\end{array}$ & $\begin{array}{l}\text { Trained with } \\
\mathbf{2 4 5} \mathbf{3 2 7}\end{array}$ & $\begin{array}{l}\text { Trained with } \\
\mathbf{4 9 0} \mathbf{6 5 3}\end{array}$ & $\begin{array}{l}\text { Trained with } \\
\mathbf{7 3 5} \mathbf{9 8 0}\end{array}$ & $\begin{array}{l}\text { Trained with } \\
\mathbf{9 0 8} \mathbf{~ 1 3 0 6}\end{array}$ & $\begin{array}{l}\text { Trained with } \\
\mathbf{1 2 2 4} \mathbf{~ x ~ 1 6 3 2}\end{array}$ \\
\hline Training & 0.75 & 2.75 & 4.50 & 9.02 & 14.55 \\
\hline $\begin{array}{l}\text { Tested on } \\
245 \times 327\end{array}$ & 0.1215 & 0.1266 & 0.1499 & 0.1219 & 0.1200 \\
\hline $\begin{array}{l}\text { Tested on } \\
490 \times 653\end{array}$ & 0.3283 & 0.3309 & 0.3886 & 0.3312 & 0.3324 \\
\hline $\begin{array}{l}\text { Tested on } \\
735 \times 980\end{array}$ & 0.8265 & 0.7521 & 0.7443 & 0.7450 & 0.7495 \\
\hline $\begin{array}{l}\text { Tested on } \\
908 \text { x 1306 }\end{array}$ & 1.3132 & 1.3333 & 1.3286 & 1.3361 & 1.3215 \\
\hline $\begin{array}{l}\text { Tested on } \\
1224 \text { x 1632 }\end{array}$ & 2.0626 & 2.0481 & 2.0654 & 2.1134 & 2.0729 \\
\hline $\begin{array}{l}\text { Tested on } \\
2448 \times 3264\end{array}$ & 8.6248 & 8.5331 & 8.5869 & 8.6438 & 8.6576 \\
\hline
\end{tabular}

\section{$Q R$ code readability}

Another experiment was conducted for QR code decoding with images in different types. 20 images were taken of a QR code from different angles. The images were converted from RGB to grayscale and binary images, using the global method (or Otsu's method) and adaptive method (or Bradley's method). As shown in Table 3, according to the experiment results, QR codes on RGB and Grayscale images have less chance of being read (30\%) than those on binary images. Moreover, binary images generated using the global method perform better (55\%) than those using the adaptive method (40\%). Because smart phones often capture RGB images, the system converts images into binary images (using the global method) before trying to capture the image's QR code. This shows that all RGB images should be converted into binary images using the global method before QR code decoding.

Table 3. Successful QR code reading-rate against 20 images in RGB, grayscale and binary

\begin{tabular}{|l|c|c|c|c|}
\hline & RGB & Grayscale & $\begin{array}{c}\text { Binary } \\
\text { (global method) }\end{array}$ & $\begin{array}{c}\text { Binary } \\
\text { (adaptive method) }\end{array}$ \\
\hline $\begin{array}{l}\text { Number of images } \\
\text { successfully decoded }\end{array}$ & 6 & 6 & 11 & 8 \\
\hline $\begin{array}{l}\text { Percentage } \\
\text { (total 20 images) }\end{array}$ & $30 \%$ & $30 \%$ & $55 \%$ & $40 \%$ \\
\hline
\end{tabular}


The empirical study has verified that the design of the inspection-repair system is workable. The classifier maintained a high accuracy (98\% to $100 \%)$ and required minimal time $(0.12$ seconds per image) with low resolution images $(245 \times 327)$. The system stores all the information in digital form and transfers the information internal between users. User interfaces function properly, supporting effective cooperation among users involved in the process. Each objective mentioned in the Introduction section of this paper was fulfilled, and the prototype is considered satisfactory.

The prototype was demonstrated to academics and employees of an industry involved in FM, who provided positive feedback and agreed that this system would greatly assist FM processes. Nevertheless, they noted that the system would not be practical unless it allowed users to access the BIM knowledge repository from different computers over the internet. In addition, a mobile phone application would be preferable in order to simplify the image uploading process and automatic image classification mechanism.

\section{Discussions and Conclusion}

The innovation of this research is the application of QR codes and image classification using Bag of Words with BIM to facilitate the FM process. This empirical study has demonstrated that the developed system has improved the information flow in the inspection-repair process by enabling users to collaborate effectively using GUIs with the same 3D model when the information is accessed automatically from and stored in the BIM knowledge repository. All records of activity, images and comments are stored in the knowledge repository. This reduces information loss while enabling personnel to review these records at any time in the future. These records enable personnel, especially repairmen, to improve their understanding of the history of an object, facilitating their activities. It is concluded that the image classification algorithms and the use of QR codes reduce the amount of time consumed by the automation of the process.

Results of this research align with findings by Lavikka et al. (2017), demonstrating that QR codes can be used in FM to enable fast information exchange and seamless cooperation. This study confirms findings by Lin et al. (2014) that two-dimensional QR codes can be used in FM to improve data accuracy and inspectionrepair processes. In order to improve the performance of QR codes, pre-processed is required before decoding. This study demonstrates that using image classification could improve the robustness of the system. With low resolution images, high accuracy can be assured in short processing times. Using QR codes and image classification, the system can reduce the time required for manual data input, improve information access and data accuracy, as well as enhancing collaboration between personnel involved in FM activities. The automated inspection-repair system developed implies that FM efficiency and data collection accuracy could be improved and costs of FM and human errors reduced. The system bridges the gap that is missing in the automated system for FM. The system developed can be applied to other industries, such road systems, where it could be used to detect infrastructure errors and maintenance.

Some limitations of this system have been noticed. If two identical objects are located in different places, the QR-code-decoding method can read the location directly from the code. However, the image classification will have difficulty identifying these items since it classifies images solely based on their appearance and features. This implies that, for example, two identical microwaves would be difficult to differentiate based on their images. Thus, further research is needed to classify objects as identical or similar. Also, although the system improves FM processes, the precise extent of improvement in terms of time and cost is unclear. Further research and experiments are required for justification.

To extend the usefulness of the system, it can be further automated by combining other technology. For instance, instead of having humans inspect buildings, images can be captured by robots that periodically patrol the facility. Alternatively, fixed RGB sensors in the corners of rooms would enable inspection without human involvement. However, sensors would need to cover all objects to be inspected, which could be costly. However, some image processing techniques may be able to detect faults and damages on objects automatically, such as comparing old images of objects with new ones, assisting in the decision-making process for facility managers. 
This research has addressed a significant problem: the current inefficient manual inspection-repair process. The study has demonstrated the design of a system that uses BIM and image classification to improve information flow in the inspection-repair process, which is a significant aspect of FM. As demonstrated in this empirical study, the system allows users to cooperate effectively with just one 3D model and to transfer information entirely in digital form, avoiding the possibility of human-error. By applying QR codes and image classification using Bag of Words, the information collection process is simplified and accelerated. Because this system is in its prototype stage, improvements will need to be made. The extent of improvement in terms of effectiveness and cost in comparison with the traditional method will need to be justified by future studies. By combining other technologies, automation of this system may be extended further.

\section{References}

Abdelfattah, A. (2017), "Image Classification using Deep Neural Networks-A beginner friendly approach using TensorFlow", available at: https://medium.com/@tifa2up/image-classification-using-deep-neural-networks-a-beginner-friendly-approachusing-tensorflow-94b0a090ccd4 (accessed 18 August 2017).

Akcamete, A., Akinci, B. and Garrett, J. (2010), "Potential utilization of building information models for planning maintenance activities", Proceedings of International Conference on Computing in Civil and Building Engineering, Nottingham University Press, Nottingham, UK.

Alfed, N., Khelifi, F. and Bouridane, A. (2016), "Improving a bag of words approach for skin cancer detection in dermoscopic images", International Conference on Control, Decision and Information Technologies (CoDIT), pp. 24-27.

Ali, N. M., Jun, S.W., Karis, M.S., Ghazaly, M.M. and Aras, S.M. (2016), “Object classification and recognition using Bag-ofWords (BoW) model”, IEEE 12th International Colloquium on Signal Processing \& Its Applications (CSPA), pp. 216-20.

Anwar, H., Zambanini, S. and Kampel, M. (2013), “A Bag of Visual Words Approach for Symbols-Based Coarse-Grained Ancient Coin Classification", OAGM/AAPR 2013 proceedings.

Aziz, N.D., Nawawi, A.H. and Ariff, N.R. (2016), "Building information modelling (BIM) in facilities management: opportunities to be considered by facility managers", Procedia - Social and Behavioural Sciences, Vol. 234, pp. 353-362.

Becerik-Gerber, B., Jazizadeh, F., Li, N. and Calis, G. (2012), "Application area and data requirements for BIM-enabled facilities management", Journal of Construction Engineering \& Management, Vol. 138, Issue 3, pp. 431-442.

Bell, L.C. and McCullouch, B.G. (1988), "Bar code applications in construction”, J. Constr. Eng. Manage, ASCE Vol. 114, Issue 2, pp. 263-278.

Bell, L. and Williams, B. (2003), "Resources and field technology for sign management system implementation", in Proceedings of Construction Research Congress 2003, ASCE, March 19-21, Held in Honolulu, Hawaii.

Bhatla, A., Choe, S.Y., Fierro, O. and Leite, F. (2012), "Evaluation of accuracy of as-built 3D modelling from photos taken by handheld digital cameras" Autom. Constr., Vol. 28, pp. 116-127.

Blakey, L.H. (1990), "Bar code: prescription for precision, performance, and productivity", J. Constr. Eng. Manage., ASCE Vol. 116 Issue 3, pp. 468-479.

Bosche, F., Ahmed, M., Turkan, Y., Haas, C.T. and Haas, R. (2015), “The value of integrating scan-to-BIM and scan-versus-BIM techniques for construction monitoring using laser scanning and BIM: The case of cylindrical MEP components", Autom. Constr., Vol. 49, pp.201-213.

Chang, Y., Chu, C. and Chen, M. (2007), “A General Scheme for Extracting QR Code from a non-uniform background in Camera Phones and Applications”, Ninth IEEE International Symposium on Multimedia (ISM 2007), pp 123-30.

Chen, C., Dib, H. and Lasker, G. (2011), "Benefits of implementing building information modelling for healthcare facility commissioning", In Proceedings of the ASCE International Workshop on Computing in Civil Engineering, Miami, FL, 1922, June, pp.578-585.

Costin, A., Shaak, A. and Teizer, J. (2013), "Development of a navigational algorithm in BIM for effective utility maintenance management of facilities equipped with passive RFID", In Proceedings of the ASCE International Workshop on Computing in Civil Engineering, Los Angeles, CA, pp. 653-660. DOI: 10.1061/9780784413029.082.

Csurka, G., Dance, C. R., Fan, L., Willamowski, J. and Bray, C. (2004), "Visual Categorization with Bags of Keypoints", in Workshop on Statistical Learning in Computer Vision, ECCV, pp. 1-22.

Dai, F., Rashidi, A., Brilakis, I. and Vela, P. (2012), “Comparison of image-based and time-of-flight-based technologies for 3D reconstruction of infrastructure”, In Proceedings of Construction Research Congress, ASCE, Reston, VA, pp. 929-939.

De Reu, J., De Smedt, P., Herremans, D., Van Meirvenne, M., Laloo, P. and De Clercq, W. (2014), "On introducing an imagebased 3D reconstruction method in archaeological excavation practice”, Journal of Archaeological Science, Vol. 41, pp. 251262 . 
Deselaers, T., Pimenidis, L. and Ney, H. (2008), "Bag-of-Visual-Words Models for Adult Image Classification and Filtering", 19th International Conference on Pattern Recognition, pp. 1-4.

Dimitrov, A. and Golparvar-Fard, M. (2014), "Vision-based material recognition for automated monitoring of construction progress and generating building information modelling from unordered site image collections", Adv. Eng. Inf., Vol. 28, Issue 1, pp. 37-49.

Ding, R. X., Du, D. K., Huang, Z. H., Li, Z. M., and Shang, K. (2015), "Variational feature representation-based classification for face recognition with single sample per person", Journal of Visual Communication and Image Representation, Vol. 30, pp. $35-45$.

Ergene, M.C. and Durdu, A. (2017), "Robotic Hand Grasping of Objects Classified by Using Support Vector Machine and Bag of Visual Words", International Artificial Intelligence and Data Processing Symposium (IDAP), pp. 1-5.

Fathi, H.I., Dai, F. and Lourakis, M. (2015), "Automated as-built 3D reconstruction of civil infrastructure using computer vision: achievements, opportunities, and challenges", Adv. Eng. Inf., Vol. 29, Issue 2, pp. 149-161.

Finch, E.F., Flanagan, R. and Marsh, L.E. (1996), "Electronic document management in construction using auto-ID", Autom. Constr. Vol. 5, Issue 4, pp. 313-321.

GS1 (2009), “QR Code Overview \& Progress of QR Code Applications”, available at: http://www.gs1jp.org/pdf/001.pdf (accessed 03 October 2017).

Gupta, V. (2017), "Image Classification using Convolutional Neural Networks in Keras", available at: https://www.learnopencv.com/image-classification-using-convolutional-neural-networks-in-keras/ (accessed 30 March 2018).

Hamiledari, H., Azar, E.R. and McCabe, B. (2018), "IFC-based development of as-built and as-is BIMs using construction and facility inspection data: site-to-BIM data transfer automation", Journal of Computing in Civil Engineering, Vol. 32, Issue 2, 04017075.

Han, D., Liu, Q. and Fan, W. (2017), "A new image classification method using CNN transfer learning and web data augmentation", Expert Systems with Applications, Vol. 96, pp. 43-56.

Hannat, M., Zrira, N., Raoui, Y. and Bouyakhf E.H. (2016), "A fast object recognition and categorization technique for robot grasping using the visual bag of words", 5th International Conference on Multimedia Computing and Systems, pp. 173-8.

He, J., Xie, Y., Luan, X., Niu, X. and Zhang, X. (2016), "A TV logo detection and recognition method based on SURF feature and bag-of-words model”, 2nd IEEE International Conference on Computer and Communications (ICCC), pp. 370-4.

Huang, H. C., Chang, F. C., and Fang, W. C. (2011), "Reversible data hiding with histogram-based difference expansion for QR code applications", IEEE Transactions on Consumer Electronics, 57(2).

Ionescu, B., Benois-Pineau, J., Piatrik, T. and Quénot, G. (2014), Fusion in Computer Vision, Springer International Publishing, Switzerland.

Kang, T.W. and Choi, H.S. (2015), "BIM perspective definition metadata for interworking facility management data", Advanced Engineering Informatics, 29:958-970. DOI: 10.1016/j.aei.2015.09.004.

Klein, L., Li, N. and Becerik-Gerber, B. (2012), "Imaged-based verification of as-built documentation of operational buildings", Autom. Constr., Vol. 21, pp. 161-171.

Lavikka, R.H., Lehtinen, T. and Hall, D. (2017), “Co-creating digital services with and for facilities management”, Facilities, Vol. 35, Issue 9/10, pp. 543-556.

Lazebnik, S., Schmid, C. and Ponce, J. (2006), "Beyond bags of features: spatial pyramid matching for recognizing natural scene categories", Proc., IEEE Conf. Computer Vision and Pattern Recognition, Vol. 2, IEEE, New York, pp. 2169-2178.

Lin, Y. C., Cheung, W. F., and Siao, F. C. (2014). "Developing mobile 2D barcode/RFID-based maintenance management system", Autom. Constr., Vol. 37, pp. 110-121.

Liu, R. and Issa, R.R.A. (2014), "Design for maintenance accessibility using BIM tools", Facilities, Vol. 32 Issue 3/4, pp. 153-159.

Liu, R. and Issa, R.R.A. (2016), "Survey: common knowledge in BIM for facility", Journal of Performance of Constructed Facilities, Vol. 30, Issue 3, 04015033.

Lorenzo, T.M., Benedetta, B., Cassano, M. and Davide, T. (2014), "BIM and QR-code. A synergic application in construction site management", Procedia Engineering, Vol. 85, pp. 520-528.

Lu, Q. and Lee, S. (2017), "Image-based technologies for constructing as-is building information models for existing buildings", Journal of Computing in Civil Engineering, Vol. 31, Issue 4, 04017005.

Meadati, P. (2016), "Automation of Information Flow between BIM and QR Code", International Journal on Recent and Innovation Trends in Computing and Communication, Vol. 4, Issue 11, pp. 219-222.

MathWorks (n.d.), "Image classification with bag of visual words", available at: http://au.mathworks.com/help/vision/ug/imageclassification-with-bag-of-visual-words.html (accessed 12 December 2016). 
McCullouch, B.G. and Lueprasert, K. (1994), “2D bar-code applications in construction”, J. Constr. Eng. Manage. Vol. 120, Issue 4, pp. 739-752.

Motawa, I. and Almarshad, A. (2013), “A knowledge-based BIM system for building maintenance”, Autom. Constr., Vol. 29, pp. 173-182.

Motamedi, A., Hammad, A. Asen, Y. (2014). "Knowledge-assisted BIM-based visual analytics for failure root cause detection in facilities management”, Automation in Construction, Vol. 43, pp. 73-83.

Navon, R. and Berkovich, O. (2005), "Development and on-site evaluation of an automated materials management and control model” J. Constr. Eng. Manage. Vol. 131, Issue 12, pp. 1328-1336.

Nguyen-Hoang, M., Le, T., Ninh, V., Che, Q., Nguyen, V. and Tran, M. (2017), “Object retrieval in past video using bag-of-words model”, International Conference on Control, Automation and Information Sciences (ICCAIS), pp 145-50.

Nowak, E., Jurie, F., and Triggs, B. (2006), “Sampling Strategies for Bag-of-Features Image Classification”, ECCV 2006, pp. 490503.

Panchal, P. M., Panchal, S. R. and Shah, S. K. (2013), “A Comparison of SIFT and SURF”, International Journal of Innovative Research in Computer and Communication Engineering, Vol. 1, Issue 2, pp. 323-7.

Panyindee, C. and Pintavirooj, C. (2013), “QR Codes Application for Reversible Watermarking Algorithm in Biomedical Images", The 6th 2013 Biomedical Engineering International Conference, pp. 1-4.

Park, J., Cai, H., and Perissin, D. (2018). "Brining information to the field: automated photo registration and 4D BIM", Journal of Computing in Civil Engineering, Vol. 32, Issue 2: 04017084.

Parn, E.A., Edwards, D.J. and Sing M.C.P. (2016), "The building information modelling trajectory in facilities managements", Autom. Constr., Vol. 75, pp. 45-45.

Parn, E.A., and Edwards, D. J. (2017), Conceptualizing the FinDD API plug-in: a study of BIM-FM integration. Autom. Constr., Vol. 80, pp. 11-21.

Pishdad-Bozorgi, P. (2017), Future smart facilities: state-of-the-art BIM-enabled facility management. Journal of Construction Engineering and Management, Vol. 143(9), pp. 02517006.

Saeed, G., Brown, A., Knight, M. and Winchester, M. (2010), "Delivery of pedestrian real-time location and routing information to mobile architectural guide", Autom. Constr., Vol. 19, Issue 4, pp. 502-517.

Santos, R., Costa A.A. and Grilo, A. (2017), "Bibliometric analysis and review of building information modelling literature publish between 2005 and 2015", Autom. Constr., Vol. 80, pp. 118-136.

Shao, H., Svoboda, T., Tuytelaars, T. and Van Gool, L. (2003), "HPAT indexing for fast object/scene recognition based on local appearance", Proc., Int. Conf. on Image and Video Retrieval, Springer, Berlin, pp. 71-80.

Shehab, T. and Moselhi, O. (2005), "An automated barcode system for tracking and control of engineering deliverables", in Proceeding of Construction Research Congress 2005, April 5-7, Held in San Diego, CA.

Stukhart, G. and Cook, E.L. (1990), "Bar-code standardization in industrial construction”, J. Constr. Eng. Manage., Vol. 116, Issue 3, pp. 416-431.

Suleiman, W., Favier, E. and Joliveau, T. (2011), "Buildings recognition and camera localization using image texture description", Proc., $\quad$ SAGEO 2011. Accessed at https://www.researchgate.net/publication/215999850_Buildings_Recognition_and_Camera_Localization_Using_Image_Textur e Description on 8 Jan. 2018.

Su, C., Chang, C., Chen, C. and Tu, C. (2014), “QR codes \& GPS functions - New applications in TAIWAN", International Conference on Machine Learning and Cybernetics, pp. 638-42.

Takran, N. and Ylmaz, R. (2015), Handbook of Research on Effective Advertising Strategies in the Social Media Age, IGI Global, USA.

Unlu, E., Zenou, E. and Riviere, N. (2017), “Ordered minimum distance bag-of-words approach for aerial object identification", 14th IEEE International Conference on Advanced Video and Signal Based Surveillance (AVSS), pp. 1-6.

Volk, R., Stengel, J. and Schultmann, F. (2014), "Building information modelling (BIM) for existing buildings: literature review and future needs", Autom. Constr., Vol. 38, pp.109-127. DOI: 10.1016/j.autcon.2013.10.023.

Vyas, K., Vora, Y. and Vastani, R. (2016), "Using Bag of Visual Words and Spatial Pyramid Matching for Object Classification along with Applications for RIS”, Procedia Computer Science, Vol. 89, pp.457-64.

Wakisaka, T., Furuya, N., Inoue, Y. and Shiokawa, T. (2000), “Automated construction system for high-rise reinforced concrete buildings", Autom. Constr. Vol. 9 Issue 3, pp. 229-250.

Wetzel E.M. and Thabet W.Y. (2015), "The use of a BIM-based framework to support safe facility management processes", Autom. Constr., Vol. 60, pp. 12-24. 
Wu J. (2012) Advances in K-means Clustering: A Data Mining Thinking, Springer, Berlin, Heidelberg.

1

2

3

4

5

6

7

8

9

10

11

12

13

14

15

16

17

18

19

20

21

22

23

24

25

26

27

28

29

30

31

32

33

34

35

36

37

38

39

40

41

42

43

44

45

46

47

48

49

50

51

52

53

54

55

56

57

58

59

60

Zhang, T., Tao, D., Li, X. and Yang, J. (2009), "Patch alignment for dimensionality reduction" IEEE Trans. Knowl. Data Eng, Vol. 21 Issue 9, pp. 1299-1313. 